\title{
AVALIAÇÃO DO CONTROLE GLICÊMICO E PONDERAL EM GRANDES OBESOS DIABÉTICOS: UM ESTUDO COMPARATIVO DA INTERVENÇÃO CLÍNICA E CIRÚRGICA
}

\author{
EVALUATION OF GLYCEMIC AND PONDERAL CONTROL IN BIG OBESES WITH DIABETICS: \\ A COMPARATIVE STUDY OF CLINICALAND SURGICAL INTERVENTION
}

\author{
Joana Cunha da Silva ${ }^{1}$, Karina Severiano Martins ${ }^{1}$, Suellen Ribeiro de Oliveira Wilken ${ }^{1}$, Roberta Peixoto Gava ${ }^{1}$, Rodrigo \\ Amaral Rios ${ }^{2}$. \\ 1- Alunas de graduação em Medicina, Faculdade de Medicina de Campos, RJ. \\ 2- Orientador e Professor da disciplina de Cirurgia da Faculdade de Medicina de campos, RJ.
}

\section{RESUMO}

O Diabetes Mellitus do tipo 2 é um transtorno metabólico caracterizado por hiperglicemia crônica de elevada morbimortalidade. O objetivo do estudo foi comparar o controle glicêmico do Diabetes Mellitus do tipo 2 por meio da intervenção do tratamento clínico e da cirurgia bariátrica, em 20 pacientes, de ambos os sexos e idade na faixa entre 20 a 65 anos, com IMC $\geq$ 35 kg/m², em Campos dos Goytacazes, RJ, em 2016. Para isso, foram analisadas a resposta ao controle glicêmico, laboratorialmente, com seguimento mínimo de 3 meses. Resultados: Do total $(n=20)$ de ambos os grupos, observou-se que a maioria são mulheres (65\%) e a maioria dos pacientes situaram-se entre 24 a 65 anos. Em relação à avaliação da glicemia de jejum, todos os pacientes do grupo cirúrgico conseguiram reduzir o nível glicêmico para abaixo de $126 \mathrm{mg} / \mathrm{dl}$ na pós intervenção, em contrapartida aos $60 \%$ no grupo clínico. Observou-se que $88,9 \%$ dos pacientes do grupo cirúrgico tiveram uma melhora dos níveis de HbA1c ( $<7 \%$ ), em contrapartida, $50 \%$ dos pacientes do grupo clínico mantiveram valores acima do ideal $(\geq 7 \%)$ após o retorno de 3 meses. Observou-se uma redução do IMC do grupo cirúrgico (média IMC $=35,2 \mathrm{~kg} / \mathrm{m}^{2} \pm$ 1,1 ), seguindo uma média de IMC de $37,7 \mathrm{~kg} / \mathrm{m}^{2} \pm 3,8$ do grupo clínico após retorno. $O$ grupo cirúrgico obteve melhor controle ponderal e da glicemia na pósintervenção do que o grupo clínico, ao observar os parâmetros de: glicemia em jejum, HbA1C e IMC.

Palavras-chaves: obesidade; diabetes mellitus tipo 2; tratamento.

\begin{abstract}
Type 2 Diabetes Mellitus is a metabolic disorder characterized by chronic hyperglycemia of high morbidity and mortality. The objective of the study was to compare the glycemic control of Type 2 Diabetes Mellitus through the intervention of clinical treatment and after bariatric surgery in 20 patients of both sexes and age in the range between 20 and 65 years, with BMI $\geq 35 \mathrm{~kg} / \mathrm{m}^{2}$, in Campos dos Goytacazes, RJ, in 2016. For this, the response to glycemic control was analyzed, with a minimum follow-up of 3 months. Results: Of the total $(n=20)$ of both groups, it was observed that the majority were women $(65 \%)$ and most of the patients were between 24 and 65 years old. In relation to fasting glucose levels, $100 \%$ of the patients in the surgical group were able to reduce the glycemic level to below $126 \mathrm{mg} / \mathrm{dl}$ in the postintervention, in contrast to $60 \%$ in the clinical group. It was observed that $88.9 \%$ of the patients in the surgical group had an improvement in HbA1c levels $(<7 \%)$; in contrast, $50 \%$ of the patients in the clinical group maintained values above the ideal $(\geq 7 \%)$ after the 3month return. A BMI reduction of the surgical group (mean BMI $=35.2 \mathrm{~kg} / \mathrm{m}^{2} \pm 1.1$ ) was observed, following a mean BMI of $37.7 \mathrm{~kg} / \mathrm{m}^{2} \pm 3.8$ of the clinical group after return. Weight and blood glucose control were better in the surgical group than the clinical group after the intervention when fasting glucose levels, HbA1C and BMI were analyzed.

Key-words: obesity; Type 2 diabetes mellitus; Treatment.
\end{abstract}




\section{INTRODUÇÃO}

A obesidade é uma doença crônica, multifatorial, que se caracteriza pelo acúmulo anormal ou excessivo de gordura no organismo, resultante de uma ingestão excessiva de calorias ou gastos reduzidos, estando associada a fatores dietéticos, educacionais, sociais, entre outros ${ }^{1}$.

A crescente incidência de obesidade e diabetes mellitus tipo 2 (DM2) em todo o mundo tem se tornado um importante problema de saúde pública. Estima-se que em 2030 a quantidade de indivíduos com diabetes mellitus chegue a 300 milhões no mundo, principalmente nos países em desenvolvimento, onde a epidemia tem maior intensidade em grupos etários mais jovens ${ }^{2,3}$.

O DM é um transtorno metabólico caracterizado por hiperglicemia crônica que está associada com dano e insuficiência de vários órgãos. A evolução da doença é a causa mais comum de cegueira, amputações e doença renal crônica, além de aumentar a incidência de infarto agudo do miocárdio e acidente vascular cerebral. Estudos epidemiológicos têm demonstrado que o excesso de peso eleva o risco de ocorrência do DM2 em 10 vezes ${ }^{3,4}$.

O objetivo do tratamento clínico-farmacológico atual do diabetes mellitus tipo 2 tem como meta a normoglicemia e segundo tendência mais recente a hemoglobina glicada $(\mathrm{HbA} 1 \mathrm{c})<7 \%$. Além disso, deve dispor de boas estratégias para a sua manutenção a longo prazo em concordância com a redução da hiperglicemia, hipertensão, dislipidemia, e outros fatores de risco cardiovascular. Neste contexto, a Sociedade Brasileira de Diabetes (SBD 2015/2016), recomenda uma abordagem que inclui modificações adequadas no estilo de vida (educação em saúde, alimentação e atividade física) associada a um agente antidiabético oral. No caso dos pacientes obesos estão indicados medicamentos que não promovam aumento na secreção de insulina e que não favoreçam o ganho de peso $^{5}$.

A cirurgia bariátrica é atualmente a melhor opção de tratamento para a obesidade mórbida, sendo complementar a outras terapias para o controle de peso. Atualmente, os requisitos para realização da cirurgia de acordo com a resolução do Conselho Federal de Medicina (CFM) n ${ }^{\circ} 1.766 / 05$, são ter idade superior a 18 anos, apresentar Índice de Massa Corporal (IMC) igual ou superior a $40 \mathrm{~kg} / \mathrm{m}^{2}$ ou igual ou superior a $35 \mathrm{~kg} / \mathrm{m}^{2}$ e comorbidades como diabetes, apneia do sono, hipertensão arterial, dislipidemia, doença coronariana, osteoartrites e outras. Além disso, o indivíduo deverá ter passado por outras tentativas pelos métodos convencionais (dieta e atividade física) e ter condições psicológicas de seguir a nova dieta imposta após a cirurgia 6 .

Entretanto estudos demonstram que após a gastroplastia os pacientes são beneficiados com a melhora clínica e laboratorial em 70 a $90 \%$ da população, pois com a redução da gordura visceral os pacientes se tornam menos resistentes à insulina ${ }^{7}$.

Desse modo, esse estudo tem como objetivo comparar o controle glicêmico em pacientes com DM2 por meio do tratamento clínico farmacológico e através da cirurgia bariátrica.

\section{MATERIAL E MÉTODO}

O estudo realizado foi do tipo Transversal e Descritivo. Participaram desta pesquisa 20 pacientes com diagnóstico de DM2, de ambos os sexos e idade variando na faixa entre 20 a 65 anos, com IMC maior ou igual a 35, atendidos nos ambulatórios de endocrinologia e de cirurgia bariátrica da rede privada em Campos dos Goytacazes, RJ, no ano de 2016. Do total de pacientes $(n=20), 10(50 \%)$ foram submetidos ao tratamento clínico e 10 ao tratamento cirúrgico $(50 \%)$. Foram analisadas a resposta ao controle glicêmico do DM2, através da coleta dos dados mediante um protocolo de pesquisa, avaliando diversos parâmetros laboratoriais com seguimento mínimo de 3 meses, de forma a fazer uma comparação do grupo de pacientes clínicos e cirúrgicos antes e após as intervenções médicas propostas. Como critérios de exclusão estão os pacientes que não se enquadraram no perfil, com dados laboratoriais incompletos ou com IMC $<35$, idade $<20$ ou maior que $>65$ anos. As variáveis analisadas foram: Sexo; Idade; IMC; Parâmetros laboratoriais de resposta ao tratamento dos pacientes atendidos, sendo estes: HOMA IR (frações da insulina), peptideo c, glicemia de jejum e hemoglobina glicada. A análise dos dados foi estatística, sendo feita uma associação entre os dados obtidos e calculada a frequência das variáveis estudadas. Todos os participantes deste estudo assinaram um termo de consentimento livre e esclarecido para a obtenção dos dados. Este trabalho obteve aprovação do comitê de ética em pesquisa.

\section{RESULTADOS}

Participaram desse estudo 20 pacientes. Destes, 10 foram submetidos a tratamento clínico para controle do Diabetes e 10 foram submetidos ao tratamento cirúrgico. Do total $(n=20)$ de ambos os grupos, observou-se que a grande maioria são mulheres (65\%). 
Em relação à idade, a maioria dos pacientes situou-se entre 24 a 65 anos de idade.

Um dos parâmetros de avaliação da resposta ao controle glicêmico do DM2 foi a glicemia de jejum.

A Tabela 1 mostra os valores para os pacientes submetidos ao tratamento clínico e cirúrgico antes e após 3 meses de retorno ambulatorial. Observou-se que a maioria dos pacientes de ambos os grupos estavam com valores acima do desejável de glicemia $(\geq 126 \mathrm{mg} / \mathrm{dl})$ pré intervenção. Entretanto, os pacientes do grupo cirúrgico obtiveram um melhor controle da glicemia na pós-intervenção, pois em nenhum destes apresentaram um nível glicêmico $\geq 126 \mathrm{mg} / \mathrm{dl}$, comparando com $40 \%$ dos pacientes do seguimento clínico. Observa-se ainda, dentro deste grupo, que metade dos pacientes conseguiram manter o nível glicêmico ideal $(<126 \mathrm{mg} / \mathrm{dl})$.

Outra maneira de se avaliar o controle glicêmico dos pacientes obesos e diabéticos é através da análise da hemoglobina glicada (HbA1c). Deste modo, a Tabela 2 faz uma comparação entre pacientes submetidos ao tratamento clínico e cirúrgico. Sendo

Tabela 1. Valores de glicemia de jejum pré e pós seguimento nos pacientes do grupo clinico versus grupo cirúrgico.

\begin{tabular}{|cccccc|}
\hline & $\begin{array}{c}\text { Glicose } \\
\text { Jejum }\end{array}$ & Pré $(\mathbf{n})$ & $\%$ & Pós $(\mathbf{n})$ & $\%$ \\
\hline Grupo & $=126 \mathrm{mg} / \mathrm{dl}$ & 7 & 70 & 4 & 40 \\
\hline clinico & $\begin{array}{c}100-125 \\
\mathrm{mg} / \mathrm{dl}\end{array}$ & 3 & 30 & 4 & 40 \\
& $\begin{array}{c}126 \mathrm{mg} / \mathrm{dl} \\
\text { Grupo }\end{array}$ & - & & 2 & 20 \\
\hline cirúrgico & $\begin{array}{c}\text { Total } \\
\end{array}$ & 10 & 100 & 10 & 100 \\
& $\begin{array}{c}100-125 \mathrm{mg} / \mathrm{dl} \\
\mathrm{mg} / \mathrm{dl}\end{array}$ & 8 & 80 & - & - \\
& $<126 \mathrm{mg} / \mathrm{dl}$ & - & 20 & 5 & 50 \\
& Total & 10 & 100 & 10 & 100 \\
\hline
\end{tabular}

Tabela 2. Avaliaçäo dos valores de $\mathrm{HbA} 1 \mathrm{C}$ antes e após o tratamento clinico ou cirúrgico dos pacientes com DM2.

\begin{tabular}{|c|c|c|c|c|c|c|c|c|}
\hline HbA1C & Fase Pré & & & $\begin{array}{l}\text { Fase } \\
\text { Pós }\end{array}$ & & & & \\
\hline & Cirúrgico & $\%$ & Clinico & $\%$ & Cirúrgico & $\%$ & Clínico & $\%$ \\
\hline$=7 \%$ & 10 & 100 & 7 & 70 & 1 & 11,1 & 5 & 50 \\
\hline$<7 \%$ & - & - & 3 & 30 & 9 & 88.9 & 5 & 50 \\
\hline Total & 10 & 100 & 10 & 100 & 10 & 100 & 10 & 100 \\
\hline
\end{tabular}


assim, todos os pacientes do grupo cirúrgico, antes do procedimento, possuíam HbA1c acima dos valores desejados $(\geq 7 \%)$ e que, após a cirurgia, $88,9 \%$ dos pacientes tiveram uma melhora dos níveis de HbA1C $(<7 \%)$. Em contrapartida, nos pacientes do grupo clínico, dos sete pacientes (70\%) com valores acima do ideal de hemoglobina glicada $(\geq 7 \%)$, cinco $(50 \%)$ mantiveram-se após o retorno de 3 meses, dentro da mesma faixa $(\geq 7 \%)$. Isto poderia ser justificado pela má adesão ao tratamento, ou ainda fatores intrínsecos do paciente.

A Tabela 3 mostra os valores da dosagem sanguínea do peptídeo $\mathrm{C}$ nos pacientes cirúrgicos. Não foi possível a dosagem do peptídeo $\mathrm{C}$ nos pacientes clínicos. Em relação aos pacientes do grupo cirúrgico, a maioria $(60 \%)$ possuía um valor de peptídeo C na faixa de $>3,5 \mathrm{ng} / \mathrm{ml}$ a após a cirurgia $90 \%$ dos pacientes obtiveram uma redução dos níveis sanguíneos.

A Tabela 4 faz uma comparação entre os valores de IMC dos pacientes dos 2 grupos antes e após a intervenção.

Observa-se uma diferença significativa na redução do IMC do grupo cirúrgico (média IMC $=35,2$ $\mathrm{kg} / \mathrm{m}^{2} \pm 1,1$ ) seguindo uma média de IMC de $37,7 \mathrm{~kg} /$ $\mathrm{m}^{2} \pm 3,8$ do grupo clínico após retorno. A maioria dos pacientes do grupo cirúrgico conseguiram sair da faixa de maior risco (grau II e III) da classificação da obesidade segundo o IMC, apenas 1 paciente mantevese com IMC $>35 \mathrm{~kg} / \mathrm{m}^{2}$, entretanto, este paciente obteve uma perda importante de massa corpórea,

Tabela 3. Avaliaçâo dos valores de peptideo $\mathrm{C}$ em pacientes do grupo cirùrgico.

\begin{tabular}{ccccc|}
\hline Peptideo C & $\begin{array}{c}\text { Antes } \\
\text { cirurgia \% }\end{array}$ & \multicolumn{3}{c}{$\begin{array}{c}\text { Após } \\
\text { cirurgia \% }\end{array}$} \\
\hline$<1,1 \mathrm{ng} / \mathrm{ml}$ & 1 & 10 & 1 & 10 \\
\hline $\begin{array}{c}1,1-3,5 \\
\mathrm{ng} / \mathrm{ml}\end{array}$ & 3 & 30 & 8 & 80 \\
$>\mathbf{3 , 5} \mathrm{ng} / \mathrm{ml}$ & 6 & 60 & 1 & 10 \\
Total & 10 & 100 & 10 & 100 \\
\hline
\end{tabular}

Tabela 4. Comparação dos valores de IMC entre pacientes do grupo clinico e cirúrgico antes e após o seguimento.

\begin{tabular}{ccccc}
\hline IMC $\left(\mathrm{Kg} / \mathrm{m}^{2}\right)$ & & & & \\
\hline & Clinico & & Cirúrgico & \\
Pacientes(n) & Antes & Após & Antos & Após \\
\hline 1 & 38,1 & 38,4 & 52,0 & 35,9 \\
$\mathbf{2}$ & 37,7 & 35,1 & 44,5 & 31,0 \\
3 & 37,9 & 37,0 & 35,5 & 25,1 \\
4 & 40,5 & 39,4 & 39,2 & 29,7 \\
5 & 35,4 & 30,0 & 44,4 & 32,8 \\
6 & 36,2 & 35,4 & 35,6 & 26,9 \\
7 & 36,6 & 37,0 & 47,0 & 34,0 \\
8 & 39,3 & 39,1 & 40,0 & 32,9 \\
9 & 42,4 & 43,2 & 36,3 & 30,1 \\
10 & 42,7 & 42,3 & 46,5 & 34,4 \\
Média \pm & $38,7 \pm 2,6$ & $37,7 \pm 3,8$ & $49,3 \pm 3,9$ & $35,2 \pm 1,1$ \\
Desvio & & & & \\
padrăo & & & & \\
\hline
\end{tabular}


saindo da classificação de risco extremamente elevado, o qual contribui na redução de risco cardiovascular.

A Tabela 5 mostra os valores das frações HOMA IR dos pacientes do grupo cirúrgico antes e após o tratamento. Também não foi possível a avaliação no grupo dos pacientes clínicos. Observa-se redução de $81,3 \%$ da do valor do HOMA IR ao grupo cirúrgico, no qual nenhum paciente apresentou HOMA IR $>4,65$ e se considerarmos a associação do IMC e o HOMA-IR dos 9 pacientes com IMC > 27,5 após a intervenção cirúrgica, apenas 1 manteve-se com HOMA IR > 3,6.

\section{DISCUSSÃO}

O DM é uma doença do metabolismo intermediário, caracterizada pela ocorrência de hiperglicemia crônica do qual o diagnóstico requer a confirmação laboratorial dos níveis glicêmicos. Quatro critérios são atualmente aceitos, sendo estes: HbA1c maior ou igual a $6,5 \%$ ou glicemia de jejum maior ou igual a $126 \mathrm{mg} / \mathrm{dl}$ ou glicemia $2 \mathrm{~h}$ após teste de tolerância a glicose maior ou igual a $200 \mathrm{mg} / \mathrm{dl}$ ou glicemia casual maior ou igual a $200 \mathrm{mg} / \mathrm{dl}$ associada a sintomas de hiperglicemia. Excetuando este último, todos os demais critérios precisam ser confirmados numa segunda dosagem, na ausência de hiperglicemia inequívoca ${ }^{5}$.

A obesidade é um fator de risco importante, independente, para uma redução da sobrevida e por consequência, dentre as principais complicações está o DM2. Atualmente a classificação mais usada da obesidade é dada pelo IMC (índice de massa corporal), sendo IMC $=$ peso $(\mathrm{Kg}) /$ altura $^{2}$. A obesidade grau I é definida por um IMC $\geq 30 \mathrm{Kg} / \mathrm{m}^{2}$, grau II IMC entre 35 a $39,9 \mathrm{~kg} / \mathrm{m} 2$ e grau III e" $40 \mathrm{~kg} / \mathrm{m}^{2}$. A obesidade leve (grau I) já é considerada alto risco para problemas de saúde. O objetivo do tratamento é reduzir o peso do paciente, em pelo menos $5-10 \%$ por uma terapia multidisciplinar e incluir uma dieta hipocalórica, além de exercícios regulares e terapia comportamental e medicamentosa. Em contrapartida, a cirurgia bariátrica vem se tornando uma alternativa para a redução de peso naqueles com IMC $\geq 35 \mathrm{~kg} / \mathrm{m} 2$ com comorbidades ou IMC e" $40 \mathrm{~kg} / \mathrm{m}^{2}$. Diversos estudos têm mostrado resultados promissores no controle e melhora da glicemia em grandes obesos submetidos à cirurgia para redução de peso ${ }^{2,7-10,14-17}$.

No estudo de Greenville (1992), 165 pacientes diabéticos foram operados pelo by-pass gástrico e $83 \%$ dos pacientes permaneceram em remissão por 14 anos de follow-up ${ }^{14}$. Já no estudo SOS (Swedish Obesity Subjects-2004) que comparou um grupo de pacientes operados com um grupo de não operados, a cirurgia bariátrica foi associada com uma redução a longo prazo na mortalidade total, diminuição da incidência de diabetes, do infarto do miocárdio e do acidente vascular cerebral ${ }^{8}$. Schauer et al (2014) realizou um estudo randomizado em que os pacientes do grupo cirúrgico tiveram melhores porcentagens de redução do peso $(24,5 \pm 9,1 \%)$ no bypass e $21.1 \pm 8,3 \%$ na cirurgia de sleeve, do que no grupo clínico com uma redução de $4,2 \pm 8,3 \%{ }^{2}$.

Diante disso, observou-se que, de acordo com a tabela 1, ao avaliar o nível glicêmico como resposta ao tratamento, pode-se observar que em ambos os grupos, clínico e cirúrgico, houve uma melhora do diabetes, entretanto, ressalta-se que $100 \%$ dos pacientes do grupo cirúrgico conseguiram reduzir o nível glicêmico para abaixo de $126 \mathrm{mg} / \mathrm{dl}$, em contrapartida 
aos $60 \%$ no grupo clínico. Branco et al analisou 83 pacientes e observou que a glicemia pré-operatória variou de 125 a $500 \mathrm{mg} / \mathrm{dl}$ caindo para a variação de 76 a $120 \mathrm{mg} / \mathrm{dl}$ no pós operatório de 3 meses. Setenta e sete $(92,7 \%)$ foram submetidos à gastroplastia (Capella) e seis (7,3\%) à sleeve gástrico. Após 3 meses da operação, 92,2\% cessaram o uso de medicamentos para diabetes, sendo que nenhum permaneceu dependente de insulina no tratamento ${ }^{7}$. Schauer et al (2003) analisou pacientes obesos e DM2 submetidos a gastroenteroanastomose em y de Roux acompanhados por até 4 anos e em $83 \%$ a glicemia plasmática de jejum e as concentrações de HbA1c voltaram aos níveis normais ou acentuadamente melhoraram (17\%) em todos os pacientes ${ }^{9}$. Um estudo inglês analisou pacientes com DM2 que faziam tratamento com dieta, sulfoniloréias, metformina ou insulina e do grupo de pacientes obesos $18 \%$ obtiveram glicemia abaixo de $140 \mathrm{mg} / \mathrm{dl}$ e $13 \%$ atingiram níveis da HbA1c abaixo de 7\% 19 .

Os níveis de $\mathrm{HbA1c}$ fornecem uma indicação da concentração média de glicose no sangue durante 2 a 4 meses anteriores, sendo um dos indicadores mais aceitos de controle glicêmico a longo prazo. Segundo a Sociedade Brasileira do Diabetes (2015/2016), de modo geral, a meta da HbA1c para diabéticos é $<7 \%$. Entretanto, em casos selecionados a meta pode ser mais rígida $(<6,5 \%)^{5}$. De acordo com a tabela 2 , todos os pacientes do grupo cirúrgico, antes do procedimento, possuíam HbA1c acima dos valores desejados (e"7\%) e que, após a cirurgia, $88,9 \%$ dos pacientes tiveram uma melhora dos níveis de HbA1c $(<7 \%)$. Em contrapartida, nos pacientes do grupo clínico, $50 \%$ dos pacientes ainda mantiveram-se após o retorno de 3 meses, valores acima do ideal $(\geq 7 \%)$. Isto poderia ser justificado pela má adesão ao tratamento, ou ainda fatores intrínsecos do paciente. Neste contexto, Lee et al observou por 12 meses 85 pacientes submetidos a cirurgia bariátrica (Sleeve) e a média da HbA1c reduziu de $8,1 \%$ para $6,1 \%$. Todos os pacientes reduziram sua $\mathrm{HbA} 1 \mathrm{c}<7 \%$, sendo que 45 pacientes $(52,9 \%)$ tiveram remissão completa da HbA1c para < $6 \%, 18(21,2 \%)$ HbA1c $<6,5 \%$ e 9 pacientes $(10,6 \%)$ $<7 \%{ }^{15}$. Sendo assim, observou-se um efetivo controle glicêmico nos pacientes.

Dixon et al demonstraram que após a cirurgia, o índice de massa corporal diminuiu de $139 \mathrm{~kg}$ e 50,1 $\mathrm{kg} / \mathrm{m} 2$ para $95 \mathrm{~kg}$ e $34 \mathrm{~kg} / \mathrm{m} 2{ }^{10}$. Assim como Dan et al que analisar 40 pacientes com DM2, constatou-se uma redução significativa do IMC de 47,7 \pm 7,9 para $34,7 \pm 5,8$ após intervenção cirúrgica. Além disso, houve uma completa remissão do DM em 34 (85\%) pacientes e em 6 pacientes (15\%) ocorreu redução do numero e/ou dosagem das medicações para controlar o DM ${ }^{16}$. Em nosso estudo, o IMC reduziu de 49,3 \pm 3,9 para 35,2 $\pm 1,1$ após a cirurgia bariátrica. Enquanto o grupo clínico não houve uma redução considerável do $\operatorname{IMC}(38,7 \pm 2,6$ para 37,7 $\pm 3,8)$, permanecendo $90 \%$ dos pacientes na classificação de obesidade grau II/III. No entanto, deve-se observar os vários viés em relação ao tratamento clínico, que dependem do efetivo seguimento dos pacientes, ao uso das medicações e controle ponderal.

Através de analises dos parâmetros clínicos e laboratoriais Varaschim et al obtiveram uma média pré operatória do IMC de 39,6 kg/m2 com queda de 6,6 $\mathrm{kg} / \mathrm{m} 2$ com 60 dias de pós operatório. A glicemia média pré operatória foi de $132 \mathrm{mg} / \mathrm{dl}$ diminuindo para 91,6 $\mathrm{mg} / \mathrm{dl}$ com dois meses de pós operatório. Semelhante aconteceu com a HbA1c a qual reduziu de $7 \%$ para $5 \%$ após 60 dias da intervenção cirúrgica ${ }^{17}$.

Os achados obtidos neste estudo foram compatíveis aos referidos por outros autores quando analisados parâmetros de glicemia, HbA1c e IMC ${ }^{2,7-}$ 10,14-17. No entanto, Zeve et al e Schauer et al (2012) corroboraram a avaliação com outros parâmetros laboratoriais como peptídeo C e HOMA IR ${ }^{18,13}$.

Zeve et al analisaram 17 pacientes e após perderem $10 \%$ do peso a média do peptídeo C reduziu de 4,3 $\pm 0,4$ para $3,4 \pm 0,4$ e após 1 ano a média obtida foi de 2,5 \pm 0,3 nos pacientes submetidos a derivação gástrica em y de Roux. Foi avaliada também a média do HOMA IR que no inicio do segmento foi de $12,5 \pm$ 2,4 e reduziu para $3,1 \pm 0,4$ e $1,6 \pm 0,3$, após $10 \%$ da perda de peso e após 1 ano , respectivamente ${ }^{18}$.

O peptídeo C é uma forma de analisar a secreção endógena de insulina pelo pâncreas, um fragmento liberado quando a pró-insulina é clivada, dando origem à insulina. Devido à resistência periférica à insulina, espera-se encontrar níveis normais $(1,1$ ng a $3,5 \mathrm{ng} / \mathrm{mL}$ ) ou elevados de peptídeo $\mathrm{C}^{11}$. A tabela 3 mostra os valores da dosagem sanguínea do peptídeo C nos pacientes cirúrgicos e após a cirurgia, 90\% dos pacientes obtiveram uma redução dos níveis sanguíneos. Sendo assim, observa-se um melhor padrão na resistência periférica à insulina e melhor função pancreática destes pacientes assim como obtido por Zeve et al ${ }^{18}$.

A resistência insulínica (RI) pode ser acessada utilizando a avaliação do índice de HOMA IR. É um marcador que pode ser calculado a partir de uma única amostra de sangue obtida em jejum através das 
dosagens da insulinemia e da glicemia ([Insulinemia $\mathrm{mU} / \mathrm{ml} \times$ glicemia mmol $/ 1 / 22,5])^{5}$. O índice de HOMA IR associado a critérios clínicos como o IMC encontrou especificidade e sensibilidade de $78,7 \%$ e $84,9 \%$ como demonstrado por Stern et al. Logo, é considerado RI se HOMA-IR $>4,65$ ou quando o IMC $>27,5 \mathrm{~kg} / \mathrm{m} 2$ e HOMA-IR $>3,60^{12}$. A tabela 5 mostra que nenhum paciente cirúrgico da fase pós intervenção apresentou HOMA-IR > 4,65 e se considerarmos a associação do IMC e o HOMA-IR dos 9 pacientes com IMC > 27,5, apenas 1 manteve-se com HOMA-IR > 3,6 após a intervenção cirúrgica. Além disso, houve redução de $81,3 \%$ do HOMA-IR ao compararmos a média dos valores antes e após a cirurgia. No estudo Schauer et al (2012) acredita-se que o controle glicêmico no grupo cirúrgico ocorreu a partir da melhora da RI o que foi evidenciado com resultados mais favoráveis de HOMA IR no grupo cirúrgico do que no grupo clínico. Sendo que a melhora após a cirurgia bariátrica ocorreu com media de 3 meses após a intervenção e foram mantidos durante os 12 meses de seguimento ${ }^{13}$.

\section{CONCLUSÃO}

Concluímos, pelos dados obtidos em nosso estudo, ao comparar o controle glicêmico do Diabetes Mellitus do tipo 2 por meio da intervenção do tratamento clínico e da cirurgia bariátrica que:
- a grande maioria são mulheres (65\%), entre 24 a 65 anos de idade;

- ambos os grupos de pacientes, clinico e cirúrgico, estavam com valores acima do desejável de glicemia. $\mathrm{Na}$ pós intervenção, todos os pacientes do grupo cirúrgico conseguiram reduzir o nível glicêmico para abaixo de $126 \mathrm{mg} / \mathrm{dl}$, em contrapartida à $60 \%$ no grupo clínico;

- 88,9\% dos pacientes do grupo cirúrgico obtiveram uma melhora dos níveis de $\mathrm{HbA} 1 \mathrm{C}(<7 \%)$, em comparação com grupo clínico que obteve $50 \%$ após o retorno de 3 meses;

- No grupo cirúrgico, observou-se uma redução de $81,3 \%$ do valor do HOMA-IR, no qual nenhum paciente apresentou HOMA-IR > 4,65; em relação a dosagem do peptídeo C, $90 \%$ dos pacientes obtiveram uma redução dos níveis sanguíneos;

- Observou-se uma diferença significativa na redução do IMC do grupo cirúrgico (média IMC $=35,2 \mathrm{~kg} / \mathrm{m}^{2} \pm$ 1,1 ), seguindo uma média de IMC de $37,7 \mathrm{~kg} / \mathrm{m}^{2} \pm 3,8$ do grupo clínico na pós-intervenção.

Diante do pressuposto, observou-se que a intervenção cirúrgica obteve melhor controle ponderal e da glicemia do que o tratamento clínico farmacológico, ao observar os parâmetros de glicemia em jejum, HbA1C e IMC.

\section{REFERÊNCIAS BIBLIOGRÁFICAS}

1. Escrivão MAMS, Oliveira FLC, Taddei JAAC, Lopez FA. Obesidade exógena na infância e adolescência. Jornal de Pediatria, v.76, n.3, p.305-310, 2000.

2. Schauer PR, Bhatt DL, Kirwan JP, et al. Bariatric Surgery versus Intensive Medical Therapy for Diabetes -3-Year Outcomes. The new england journal of medicine 2014; 370:2002-13.

3. Monteiro FC, Junior WSS, Filho NS, et al. Efeito da Perda Ponderal Induzida Pela Cirurgia Bariátrica sobre a Prevalência de Síndrome Metabólica. Arq Bras Cardiol 2009; 92(6): 452-456.

4. Zeve, JLM, Tomaz, CAB. Cirurgia Metabólica - Cura para Diabetes Tipo 2. Arq Bras. Cir. Dig, 2011;24(4):312-317.

5. Diretrizes da Sociedade Brasileira de Diabetes (2015-2016). Disponível em: http://www.diabetes.org.br/profissionais/ images/docs/DIRETRIZES-SBD-2015-2016.pdf.

6. Barros LM, Frota NM, Moreira RAN, et al. Avaliação dos Resultados da Cirurgia Bariátrica. Revista Gaúcha de Enfermagem, 2015; 36(1): 21-27.

7. Branco AJ, Menacho AM, Nassif LS, Hirata LM, Gobbi RI, Perfete C, et al. Gastroplastia como tratamento do diabete melito tipo 2. Arquivo Brasileiro de Cirurgia Digestiva, 2011; 24(4):285-289.

8 . Sjostrom L. Review of the key results from the Swedish Obese Subjects (SOS) trial - a prospective controlled

intervention study of bariatric surgery. Journal of interne Medicine, 2013; 273(3):219-234.

9. Schauer PR, Burguera B, Ikramuddin S, et al. Effect of laparoscopic Roux-en Y gastric bypass on type 2 diabetes mellitus. Ann Surg, 2003; 238(4): 467-485.

10. Dixon JB, O’brien PE, Playfair J, et al. Adjustable Gastric Banding and Conventional Therapy for Type 2 Diabetes: A Randomized Controlled Trial. JAMA, 2008; 299(3): 313-316.

11. Bellini JC, Macedo PB, et al. Avaliação da secreção pancreática de insulina pela dosagem do peptídeo C. Grupo Editorial Moreira JR, 2006; 63(10):544-545.

12. Stern SE, Williams k, Ferrannini E, et al. Idetenfication of individuals with insulin resistence using routine clinical measurement. Diabetes Journal, 2005; 54(2): 333 - 339. 
13. Schauer PR, Kashyap SR, Wolski K, et al. Bariatric Surgery versus Intensive Medical Therapy in Obese Patients with Diabetes. The New England Journal of Medicine, 2012; 366(17): 1567-1576.

14. Geloneze B, Pareja JC. Cirurgia bariátrica cura a síndrome metabólica? Arquivos Brasileiros Endocrinologia e Metabologia, 2006; 50(2): 400-407.

15. Lee WJ, Almulaifi A, Tsou JJ, Ser KH, Lee YC. Laparoscopic sleeve gastrectomy for type 2 diabetes mellitus: predicting the success by ABCD score. American Society for Metabolic and Bariatric Surgery, 2015; 11(5):33-46.

15. Dan D, Harnanan D, Singh Y, Hariharan S, Naraynsingh V, Teelucksingh S. Effects of bariatric surgery on Type - 2 Diabetes Mellitus in a Caribbean setting. International Journal of Surgery, 2011; 9(5):386-391.

16. Varaschim M, Nassif PA, Moreira LB, Nascimento MM, Vieira GM, Garcia RF, et al. Alterações dos parâmetros clínicos e laboratoriais em pacientes obesos com diabetes melito tipo 2 submetidos à derivação gastrojejunal em y de Roux sem anel. Revista Colégio Brasileiro de Cirurgiões, 2012; 39 (3): 178-182.

17. Zeve JLM, Tomaz CAB, Nassif PA, Lima JH, Sansana LR, Zeve CH. Obesos diabéticos tipo 2 submetidos à derivação gástrica em Y-de-Roux: análise de resultados e influência nas complicações. Arq Bras. Cir. Dig. , 2013; 26: 47-52.

Turner RC, Cull CA, Frighi V, Holman RR. Glycemic control with diet, sulfonilureya, metformin, or insulin in patients with type 2 diabetes mellitus. JAMA, 1999; 281: 2005 - 2012. 